\section{A smooth ride for galaxies}

\author{
M. G. Edmunds \& S. Phillipps
}

SEARChes for large-scale distributions of matter in the Universe are all the rage nowadays, as Dressler indicated in a review article in last week's issue ${ }^{1}$. But the picture of how these distributions are gravitationally pulling our Galaxy and its neighbours around our corner of the Universe seems to be much simpler than has been supposed, calculations by James, Joseph and Collins suggest ${ }^{2}$. The difficulties seem to have arisen from biases inherent in the sampling necessary to make large-scale surveys manageable.

The expansion of the Universe has been known since Edwin Hubble's fundamental discovery of a regular relationship between how far away a galaxy is, and its velocity away from us. Except for the very nearest galaxies, the velocities increase almost linearly with distance. The "almost" qualification comes from the slight inevitable variation of individual galaxy velocities about the mean expansion, just as one would not expect all atoms in an expanding gas to have exactly the same speed. The basic origin of a galaxy's extra motion is the perturbing effect of the gravitational pull of the galaxies or clusters of galaxies in its neighbourhood. In recent years such motions have been intensively studied.

When considering the motion of individual galaxies relative to the general expansion, there is one fundamental reference frame the microwave background radiation left over from the early Universe, predating the development of significant deviations from a Hubble flow. The Doppler shift of the microwave background (essentially a slight temperature anisotropy) shows that own little group of galaxies (which is a few megaparsecs in size) is moving at about $600 \mathrm{~km} \mathrm{~s}^{-1}$ relative to this 'fixed' frame in a direction which, viewed from Earth, is towards the constellation Hydra.

Some years ago, before our motion relative to the microwave background had been determined, a claim was made ${ }^{3}$ that our local group showed a motion of some $450 \mathrm{~km}$ $\mathrm{s}^{-1}$ relative to moderately distant galaxies $\left(35 h^{-1}\right.$ to $65 h^{-1} \mathrm{Mpc}$ away, where $h$ is the Hubble constant in units of $100 \mathrm{~km} \mathrm{~s}^{-1}$ $\mathrm{Mpc}^{-1}$, and probably $0.5 \leqq h \leqq 1$ ). This apparent motion, known as the Rubin-Ford effect, turned out not to be in the same direction as our motion through the microwave background. Taken literally, this would imply a gross streaming motion - relative to the microwave background - of the galaxies in a very large local volume of space, some $100 \mathrm{~h}^{-1} \mathrm{Mpc}$ in size. The cause of such largescale streaming was unknown. Although it stimulated a lot of useful work, it now seems that the original Rubin-Ford effect should not be interpreted as implying a streaming motion at all. As so often in observational astronomy, it appears that the selection of the particular sample biased the results.
In a rather convincing paper, James, Joseph and Collins ${ }^{2}$ argue that the basic problem came from taking only galaxies with a restricted range of apparent brightness (as seen from Earth) and in using their exact brightness to estimate the distances of the galaxies, on the assumption that all of the particular type of galaxy used have the same intrinsic brightness. The trouble with this procedure is that real galaxies do have a distribution in intrinsic brightnesses, and the result - as discovered in surveys of stars in the 1920 s by the Swedish astronomer Malmquist - is that the distant galaxies observed in the survey tend to be intrinsically brighter than average, and the nearest ones are on average intrinsically fainter. If the distances are being deduced from these brightnesses, then the redshifts (the measure of velocity) of the nearer galaxies seem anomalously low and the redshifts of the furthest ones seem anomalously high - and the difference is attributed to a systematic motion. Combine this with differences in average distances to galaxies in different directions in the sky, attributable to the way the sample was chosen or to galaxy clustering, and an apparent flow in some direction can result.

What James, Joseph and Collins have done is to ask the question: if the sample of galaxies is really moving smoothly with the Hubble flow, and the galaxies have a reasonable intrinsic spread of brightnesses, and the galaxies are chosen as they were - on the basis of their velocities relative to us - then what sort of velocity distribution would we expect to see? From simulations they conclude that, even with smooth Hubble flow, the sample would appear to have spurious streaming flows of around $600-800 \mathrm{~km} \mathrm{~s}^{-1}$. This pleasing result brings us back to the 'simplest' picture of regular Hubble flow without additional large-scale velocity fields.

But what about the $600 \mathrm{~km} \mathrm{~s}^{-1}$ motion of our local group relative to the microwave background? The interpretation may have become considerably clearer following a survey of redshifts ${ }^{4}$ based on a catalogue of galaxies detected as infrared sources by the IRAS satellite. The claimed advantage of using this sample is that it is less prone to the systematic selection effects which can plague optical samples. Analysis of the velocities in the sample, out to about $200 h^{-1} \mathrm{Mpc}$, gives a picture of general expansion, superimposed on which are local perturbations due to the gravitational effect of 12 massive clusters or superclusters of galaxies. The $600 \mathrm{~km} \mathrm{~s}^{-1}$ of our local group is mainly due to the attracting effect of the great clusters in the direction of Virgo, Hydra-Centaurus, Pavo and Fornax-Eridanus, and whose centres are within $65 h^{-1} \mathrm{Mpc}$ of us.

The only problem is the implied extraordinary mass and size of the clusters. They must contain a great deal of dark matter (perhaps 30 times that visible as galaxies) and extend out $30 h^{-1} \mathrm{Mpc}$ from their centres - which may be compared with their 'visible' size, a few (perhaps $3 h^{-1}$ ) megaparsec. At this size, many of the big clusters essentially merge into each other. Perhaps this should not be regarded as a problem - after all, it was difficulties with the high velocity dispersions in clusters that first led Zwicky to identify the problem of 'missing mass' in the 1930s.

It now seems possible to dispense with the idea that there might be some additional source of perturbing gravitation - the socalled Hidden Great Attractor - pulling on the galaxies in our neighbourhood, the observed clusters themselves being massive enough to account fully for the local group motion. The samples ${ }^{5}$ which gave rise to the idea of a Great Attractor may also suffer from selection effects, in particular they used elliptical galaxies which biased the samples towards galaxy cluster members, as ellipticals are far more common in clusters than in the general field between clusters.

It also seems ${ }^{6}$ that the method of estimating distances to the ellipticals (crucial when their Hubble flow velocities are estimated from their distances) may involve large random and systematic errors. The distance estimator uses the fact that the (fairly easily measured) internal velocity dispersion of the stars in a galaxy is directly related to its intrinsic brightness: both essentially depend on the mass of the galaxy. But the relation seems to vary between different clusters, perhaps because of the different histories or environments of the galaxies in particular clusters, and hence cannot be used as a reliable universal indicator of distance.

Does this mean that there is no Great Attractor at all? Semantics seems to have confused the issue. The "group of seven" 1,5 still support the concept, but identify it with the mass associated with the galaxies in that part of the sky. The argument now seems to be about whether the local flow is said to be due to the mass in a particularly dense concentration, or to the combined effect of several large clusters.

The kinematics of galaxies - and of galaxy clusters - in the Universe may soon be presenting a rather more straightforward picture of expansion than was threatened a few years ago. What remains is the problem of the size and the nature of the mass in the great clusters, although the very fact of the concentration of the mass into these structures must surely provide a valuable clue. $\square$

M. G. Edmunds and S. Phillipps are in the Department of Physics, University of Wales, PO Box 913, Cardiff CF1 3TH, UK.

1. Dressler, A. Nature 350, 391-397 (1991).

2. James, P. A., Joseph, R. D. \& Collins, C. A. Mon.Not.R.astr. Soc. 248, 444-450 (1991)

3. Rubin V. C., Ford W. K., Thonnard, N., Roberts, M. S. \& Graham, J. A. Astron. J. 81, 687-697 (1976).

4. Rowan-Robinson, M. et al. Mon.Not.R.astr.Soc. 247, 1-19 (1990).

5. Lynden-Bell, D. et al. Astrophys. J. 326, 19-49 (1988).

6. Lucey, J. R., Gray, P. M., Carter, D. \& Terlevich, R. J. Mon.Not.R.astr.Soc. 248, 804-815 (1991). 